\title{
The Drosophila pourquoi-pas?/wings-down zinc finger protein: oocyte nucleus localization and embryonic requirement
}

\author{
Laurent Ségalat, Régis Perichon, Jean-Pierre Bouly, and Jean-Antoine Lepesant ${ }^{1}$ \\ Institut Jacques Monod, Centre National de la Recherche Scientifique et Université Paris 7, 2, 75251 Paris Cedex 05, France
}

The pourquoi-pas? (pqp) gene of Drosophila melanogaster encodes a $\mathrm{Cys}_{2} / \mathrm{His}_{2}$ zinc finger protein and is abundantly transcribed in adult ovaries. During oogenesis, we immunodetected the pqp protein in the nucleus of nurse cells at stages 1-6, in a spherical structure within the oocyte nucleus at stages $7-9$, and uniformly distributed in the oocyte nucleus and in nurse cell nuclei at later stages. The pqp protein is also present at a lower level in the nuclei of follicle cells, embryos, and larvae. By means of a polymerase chain reaction (PCR) screen, we recovered three independent and phenotypeless P-element insertions at the pqp locus. In a second step, two excision-induced deletions of the pqp gene were isolated after mobilization of one of these $P$ elements. The pqp mutants display zygotic (spread and drooping wings, cross-vein defects, extra bristles) and maternal (embryonic lethality) recessive phenotypes. The chromosomal position (98EF) of the pqp gene and the drooping wing phenotype of the pqp mutants agree with the hypothesis that the pqp gene is the wings down $(w d n)$ gene for which T.H. Morgan isolated (and lost) mutants in the 1920s. This is the first reported occurrence of a zinc finger protein in the nucleus of the Drosophila oocyte.

[Key Words: Drosophila; oogenesis; female sterile mutation; zinc finger protein]

Received March 2, 1992; revised version accepted March 26, 1992.

The early development of an embryo relies on maternal components of the egg until zygotic transcription fully provides for the needs of the embryo. Two groups of factors are required for proper transition from the egg to the self-sufficient embyro: One permits the embryo to develop until transcriptional activation occurs, and the other enables the embryo to develop correctly past transcriptional activation by switching on the correct combination of zygotic genes. Maternally inherited time 0 transcription factors are essential to development as they are at the top of the cascade of genetic events, which lead to determination, differentiation, and morphogenesis (Gurdon 1992; St. Johnston and NüssleinVolhard 1992).

In Drosophila, the mechanisms of zygotic transcriptional activation are not understood. The earliest zygotic transcripts are detected during the eleventh cycle of nuclear divisions, but transcription reaches a high level only during the fourteenth cycle, which is concomitant with cellularization (McKnight and Miller 1976; Edgar and Schubiger 1986). Transcription can be activated at the 10th cycle by artificially extending the cycle length (Edgar and Schubiger 1986). It has been reported recently that transcription of a reporter gene under the control of mutated $\mathrm{ftz}$ promoter sequences takes place at the third cycle (Lesley Brown et al. 1991). This demonstrates that

\footnotetext{
${ }^{1}$ Corresponding author.
}

during the first division cycles the embryo is competent for transcription, although transcription does not occur. Whether this repression acts by a generic and noninformational mechanism (Edgar and Schubiger 1986) or by specialized transcription repressors remains to be elucidated. In any case, a precise set of transcription factors is necessary to define the initial transcription pattern of the embryo.

In Drosophila, numerous maternal effect mutations leading to embryonic lethality have been reported (Schüpbach and Wieschaus 1989). It is likely that some of them correspond to genes coding for the time 0 transcription factors. On the other hand, if these genes are also required during the life cycle of the fly, their absence will lead to lethal zygotic phenotypes and they will not appear on screens for maternal effect genes.

Zinc finger proteins are potential contributors to the class of time 0 transcription factors (Evans and Hollenberg 1988). The tramtrack (ttk) zinc finger protein responsible for $f t z$ repression may be one of them (Harrison and Travers 1990). The $t t k$ mRNA is maternally provided to the embryo and disappears when $f t z$ transcription begins (Lesley Brown et al. 1991). Because no $t t k$ mutant is known, the overall $t t k$ role is unclear.

The pourquoi-pas? (pqp) gene (formerly sry $h-1$ ) could also be a member of this class of genes. It was cloned by homology to the Drosophila serendipity delta and beta zinc finger genes (Vincent et al. 1988). The pqp gene 
encodes a protein with eight zinc fingers of the $\mathrm{Cys}_{2} /$ $\mathrm{His}_{2}$ type and two acidic domains (Vincent et al. 1988). The $p q p$ mRNA is present at a high level in the ovaries of adult females, absent in males, and present at a low level in embryos and larvae. We report here the detailed expression of the pqp protein during oogenesis and the isolation of $p q p$ mutants selected after a polymerase chain reaction (PCR) screen. Results obtained by both approaches suggest that the pqp protein may be a time 0 transcription factor.

\section{Results}

\section{pqp expression}

The pqp protein is present at a high level in adult ovaries To investigate further the role of the pqp protein during oogenesis, we raised and affinity-purified anti-pqp polyclonal antibodies. In toto staining of wild-type female ovaries with these antibodies showed that the protein is first detected at stage 1 of oogenesis in the nucleus of the nurse cells. As oogenesis proceeds, the pqp protein remains located in the nucleus of the nurse cells until stage 6 (Fig. 1a). At stage 7, it is no longer detectable in the nurse cells and appears concentrated in one for occasionally two densely stained spherical structures in the oocyte nucleus (Figs. $1 \mathrm{~b}$ and $2 \mathrm{a}$ ). These structures are distinct from the nucleolus, which appears as a large smooth area not stained by anti-pqp antibodies within stage 8 oocyte nuclei (Fig. 3). This was confirmed by immunostaining of ovaries with the nucleolus-specific Ajl antibody (Saumweber et al. 1980) (data not shown). During stage 8 , the oocyte nucleus pattern remains unchanged. During stage 9, one observes a new accumulation of pqp protein in the nurse cell nuclei, which culminates at stage 10A (Fig. 1c,d) and decreases with degeneration of nurse cells in stages 11 and 12 . In the oocyte nucleus, the staining of the spheres progressively weakens during stage 9 , whereas the whole nucleus gradually becomes shaded as if the spheres melted into the nucleus (Figs. $1 \mathrm{c}$ and $2 \mathrm{~b}, \mathrm{c}$ ). This process is completed by stage 10 when the pqp protein appears uniformly distributed in the oocyte nucleus (with the exception of the nucleolus) (Figs. 1d and 2d). This staining pattern remains unchanged during later stages of oogenesis. No staining was ever detected in nurse cells or oocyte nuclei in control slides incubated without the primary antibody.

The pqp protein is also detected at a weaker level in the nucleus of both follicle and border cells (Fig. 1e). In these cells, the protein appears to be uniformly distributed throughout the nucleus.

Anti-pqp antibodies were also used to detect the pqp protein in young embryos. In toto staining done on embryos before nuclear migration to the cortex did not allow the detection of pqp protein. From the tenth nuclear division to the end of cellularization, the pqp protein was detected at a low level in all nuclei except those of pole cells (Fig. 4). The staining intensity observed in gastrulating embryos was at the level of the background. The pqp protein was detected in embryos after germ-band retraction and in larvae. Its distribution during these stages was not investigated in detail.

\section{pqp mutants}

A mutation is the most direct way to determine a gene function as the mutant's phenotype displays the consequences of the gene absence. Because the phenotype of the pqp mutation was hard to predict on the basis of the expression pattern and because no deficiency of the $98 \mathrm{E}$ region was available, we carried out an insertional mutagenesis and used a molecular screen to detect insertions of $\mathrm{P}$ transposable elements in the vicinity of or at the pqp gene (Ballinger and Benzer 1989; Kaiser and Goodwin 1990).

Recovery of P-element insertions in the 5'-flanking region of the pqp gene To generate P-element transpositions, we combined the Birmingham 2 chromosome as a source of defective P elements and the $\mathrm{P}[\mathrm{ry}+\Delta 2-3]$ (99B) source of transposase (see Materials and methods) (Engels 1988; Robertson et al. 1988). Preliminary experiments revealed that this genetic combination yields an average of four P-element insertions per third chromosome per gamete.

After mobilization of P-transposable elements, P-element insertions in or near the $p q p$ gene were detected by PCR. Primers were devised to screen both the coding and the 5 '-flanking region of the pqp gene. Of the 40,000 third chromosomes tested $(160,000$ insertions $)$, we recovered three independent insertions in the $5^{\prime}$-flanking region of the pqp gene. The position of the insertion site was deduced from the size of the amplified DNA fragment. Insertions were located at -700 (line 407), - 760 (line 106), and -810 (line 317 ) relative to the transcription initiation site (Fig. 5A,B). Chromosomes carrying the $\mathrm{P}$ insertions were recombined a wild type chromosome to separate P-element insertions at the $p q p$ locus from the others. The number of $P$ elements remaining in recombinant chromosomes was ascertained by Southern blot analysis. After several recombinations, insertions 317 and 407 were isolated. The third line (106) was not conserved as the $\mathrm{P}$ insertion in the $p q p$ locus could not be separated from another P element on the chromosome.

Deletions in the pqp gene lead to zygotic and maternal phenotypes Flies homozygous for chromosomes carrying insertions 317 and 407 presented no phenotype. A second mutagenesis was carried out to recover deletions generated by imprecise P-element excision. For that purpose, the P-element insertion 407 was mobilized by the $\mathrm{P}[r y+\Delta 2-3](99 \mathrm{~B})$ source of transposase and the progeny were tested by Southern blot analysis of individual lines. Of the 1500 lines tested, nine independent deletions were identified by causing a band shift (Fig. 5B). Seven lines gave no visible phenotype when homozygous. The other two (lines 181 and 48) exhibited similar phenotypes and did not complement.

Both lines (181 and 48) exhibit zygotic and maternal phenotypes. Three recessive zygotic $p q p$ phenotypes can 

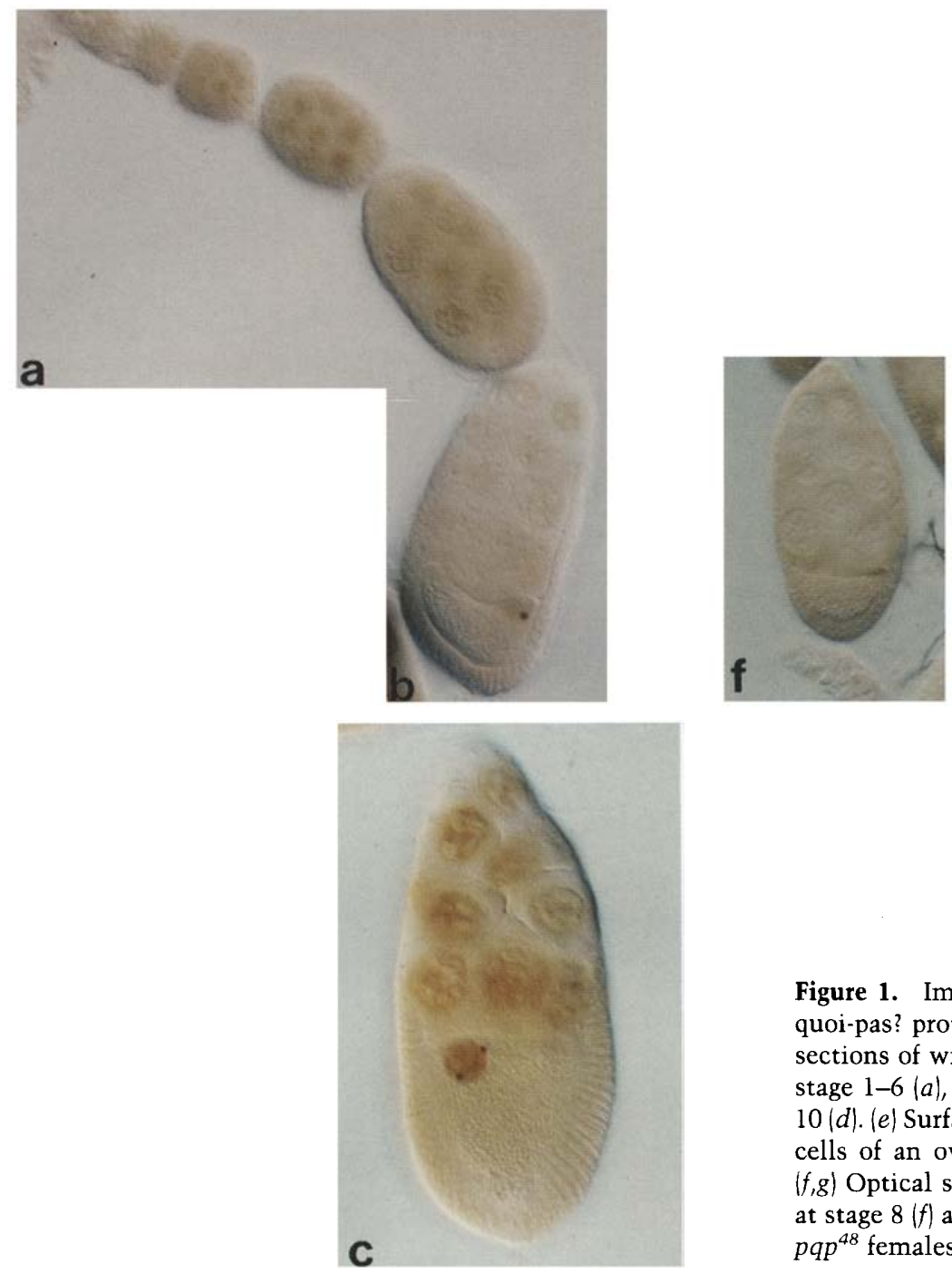

Figure 1. Immunodetection of the pourquoi-pas? protein in ovaries. $(a-e)$ Optical sections of wild-type ovarian chambers at stage 1-6 $(a)$, stage $8(b)$, stage $9(c)$, stage $10(d) .(e)$ Surface view showing the follicle cells of an ovarian chamber at stage 10 . $(f, g)$ Optical sections of ovarian chambers at stage $8(f)$ and $10(10)$ from homozygous $p q p^{48}$ females.
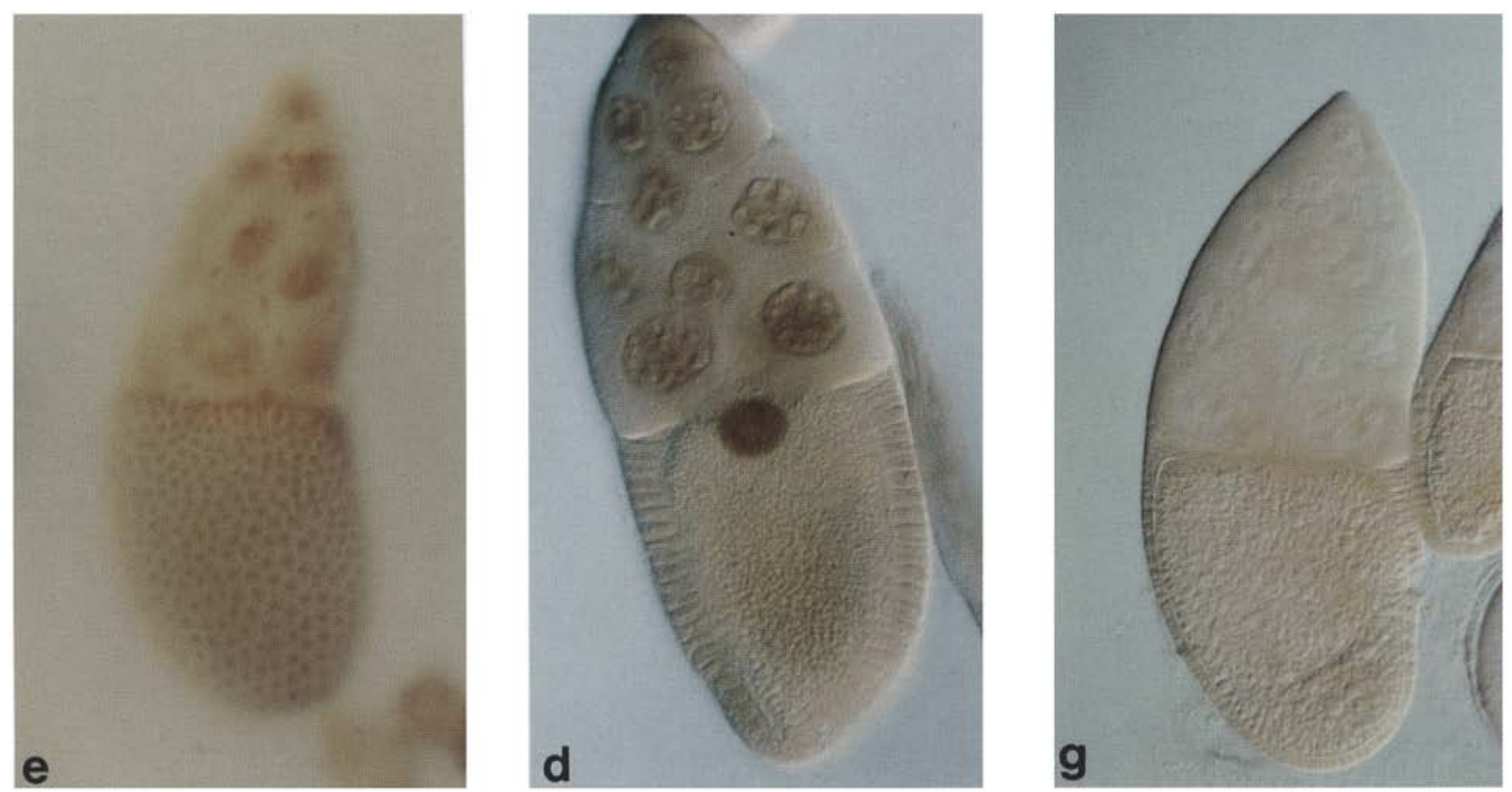
Figure 2. Immunodetection of the pourquoi-pas? protein in wild-type ovaries. Enlargement of the area of the oocyte nucleus showing the pattern transition from stages 8-10. (a) Stage 8 ovarian chamber; $(b, c)$ stage 9 ovarian chambers; $(d)$ stage 10 ovarian chamber.
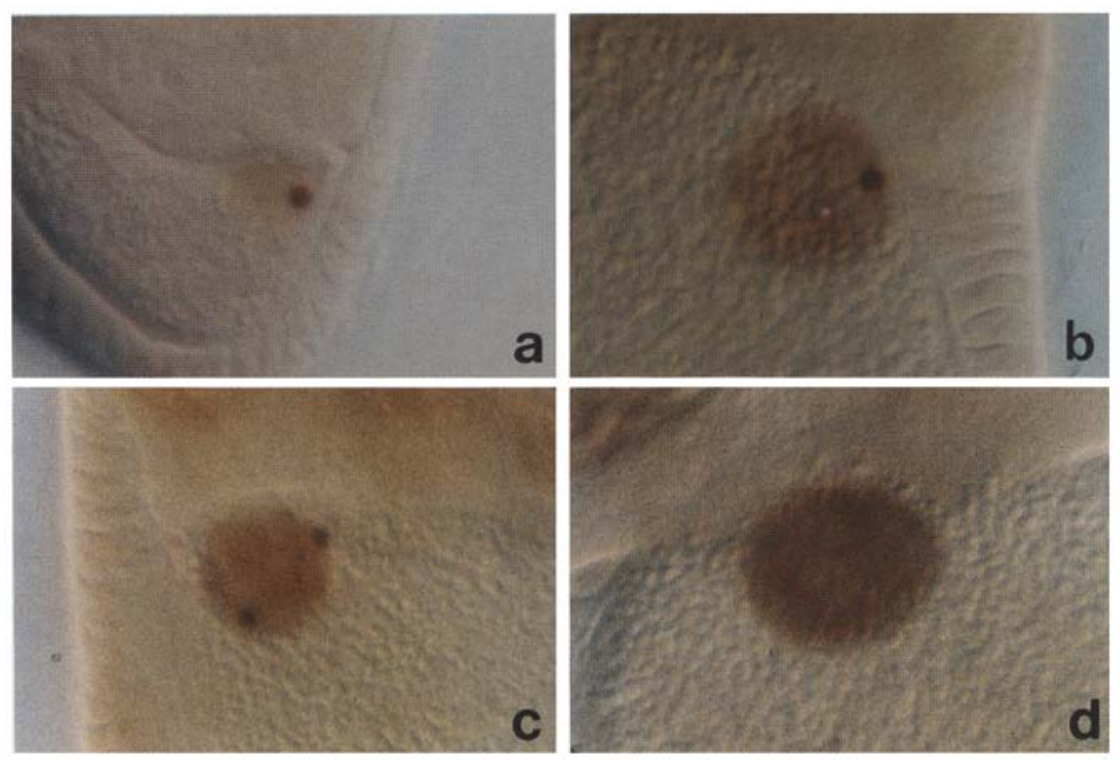

be distinguished (Fig. 6). The most striking phenotype is the position of the wings, which are spread out. Young individuals hold their wings at $\sim 45^{\circ}$ above horizontal, whereas older flies have drooping wings that drag on the medium and prevent the fly from walking normally (Fig. 6a). Some of the young flies also display this drooping phenotype. A second, not fully penetrant, phenotype consists of a double morphological defect of the wing. The anterior cross-vein is either deformed or eliminated by increased proximity of veins L 3 and L4. The posterior cross-vein is interrupted, and the anterior part is missing (Fig. 6b). The third phenotype consists of a duplication or triplication of some of the machrochaetes; the scutellar, postalar, and dorsocentral are the most frequently affected (Fig. 6c). The range of extra bristle per fly is 2 to 6 . It should be noted that the extra bristle phenotype is stronger in females than in males.

In addition to the zygotic phenotypes, there is a maternal effect phenotype. Females homozygous for one or the other deletion give no viable progeny, whatever the genotype of the mating male. No morphological defect was observed in the ovaries of these females, and the eggs they laid were morphologically normal. These eggs can be divided into three classes. Approximately $75 \%$ of the eggs appear to be unfertilized and undergo no nuclear division. Approximately $25 \%$ of them undergo nuclear cleavages and begin to cellularize. Complete cellularization takes place in most but not all of the embryos. For most of the fertilized embryos, morphological movements leading to gastrulation and germ-band extension are severely disrupted (Fig. 7). As a consequence, the body plan of these embryos is dramatically disorganized at $3 \mathrm{hr}$ after fertilization. It results in developmental defects varying from one embryo to another, but in all cases leads to embryonic lethality. Dechorionation reveals grossly abnormal shapes of these embryos (data not shown). Some of these embryos produce patches of cuticule, but no denticle belt can be recognized. One to three percent of the embryos do not show developmental alterations before and during gastrulation and germ-band extension. These embryos die as morphologically normal prehatching embryos or even hatch as apparently normal larvae. The latter die during the first or second larval stage as no third-instar larvae were recovered. This

Figure 3. Immunodetection of the pourquoi-pas? protein in wild-type ovaries. Enlargement of the area of the oocyte nucleus showing the nucleolus. Stage 8 ovarian chambers showing one $(A)$ or two $(B)$ anti-pqp detected spherical structures and the nucleolus (arrows). Bar, $10 \mu \mathrm{m}$.

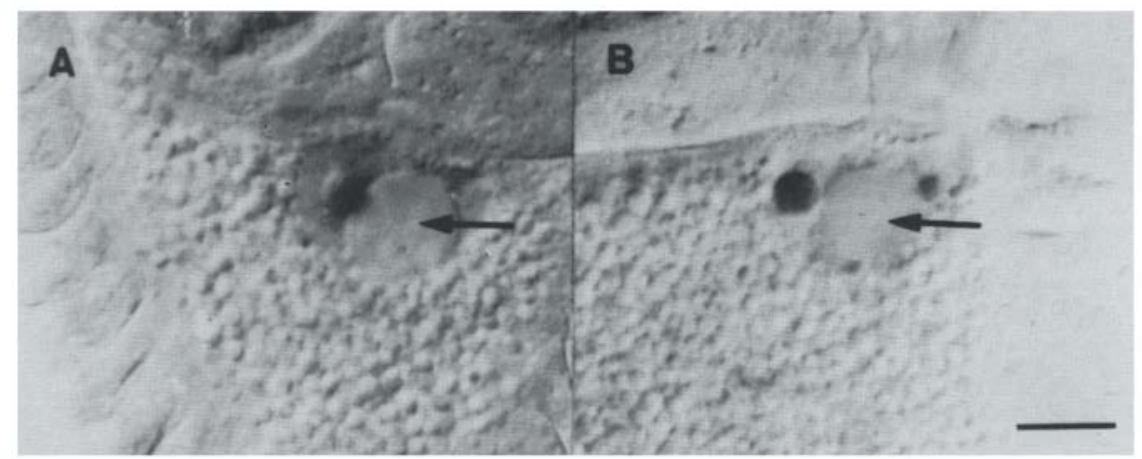




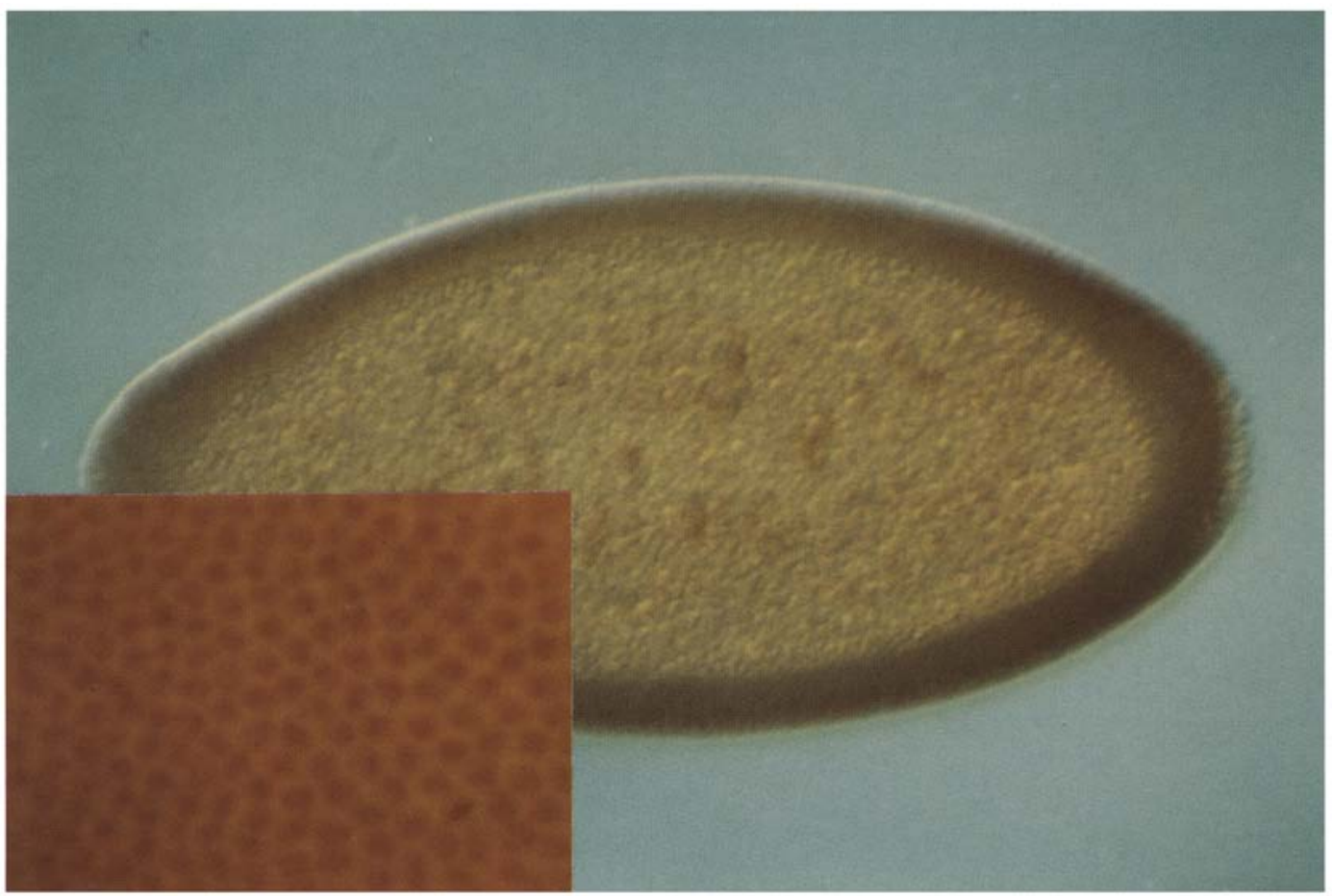

Figure 4. Immunodetection of the pqp protein in a wild-type embryo. Optical section of a cellular blastoderm embryo. (Inset) Enlargement of a top view of a cellular blastoderm embryo showing the nuclear localization of the pourquoi-pas? protein.

is a late consequence of the maternal effect, as it takes place irrespective of the genotype of the father.

pqp mutants carry deletions in the pqp gene Southern blot analysis revealed that the wild-type 2.9-kb EcoRIEcoRI $(-1700$ to +1200$)$ fragment encompassing the insertion sites was reduced in size in both mutant lines. The EcoRI-EcoRI fragment was shifted to $2.2 \mathrm{~kb}$ and 1.3 $\mathrm{kb}$ in lines 181 and 48, respectively (see Fig. 5B). Adjacent fragments were not changed, suggesting that the shift was a result of an internal deletion of the original $2.9-\mathrm{kb}$ fragment. Sequence analysis revealed that the $5^{\prime}$ breakpoint of the deletion is located at -715 in both lines. In line 181, the $3^{\prime}$ breakpoint is at position -30 (relative to the transcription start site) (see Fig. 5A). In line 48 , the $3^{\prime}$ breakpoint is at position +898 . In this line, the deletion removes 700 bp of $5^{\prime}$-flanking sequences, the transcription initiation site, the translation initiation signal, and the first quarter of the coding sequence (see Fig. 5A). Therefore, one can predict from its molecular structure that $p q p^{48}$ is a null allele of $p q p$. As $p q p^{181}$ is phenotypically indistinguishable from $p q p^{48}$, we consider that it is a null allele as well. The absence of the pqp protein in both lines was confirmed by immunohistochemistry. When incubated with anti-pqp antibodies, ovaries of homozygous females display a background level staining, indicating that the pqp protein is absent in these females (Fig. lf,g).

All pqp phenotypes can be rescued by an extra copy of the wild-type pqp gene and not by a mutated copy To confirm that the phenotype observed was a result of a lack of $p q p$ function, a phenotype rescue test was performed by introducing an extra copy of the pqp gene into transgenic flies. Two constructs were tested for rescue. In a first construct, the wild-type Sall-Sall $6.1-\mathrm{kb}$ fragment encompassing the $p q p$ locus (see Fig. 5A) was cloned in a transformation vector and introduced in the fly genome by P-element transformation. While the SalI-Sall $6.1-\mathrm{kb}$ fragment was being cloned in the transformation vector, we determined that this fragment also contained the $3^{\prime}$ end of an unidentified 4-kb transcription unit located upstream from the $p q p$ gene (Vincent et al. 1988) (see Fig. 5A). The $3^{\prime}$ end of the 4-kb transcript was mapped roughly between positions -3000 and -1700 relative to the $p q p$ transcription initiation site, at least $1000 \mathrm{bp}$ upstream from the deletion breakpoint in the mutant lines. Only a fraction of this transcript was comprised in the SalI-SalI fragment, but we wanted to ascertain that the phenotype rescuing activity relied only on $p q p$ function. For that purpose, a second (control) construct was made in which the 6.1-kb SalI-Sall fragment was mutated so as to delete bases +1 to +300 of the pqp gene (comprising the 5 '-transcribed untranslated region plus the first 20 amino acids). The mutated SallSall fragment was cloned in the same transformation vector as the wild type and introduced into the fly genome. Two transgenic lines were examined for each construct. The wild-type copy of each line rescued all maternal and zygotic phenotypes, and the mutated copy rescued none.

The -280 to -30 region is necessary for pqp expression, whereas the -700 to -280 region is dispensable The 
A
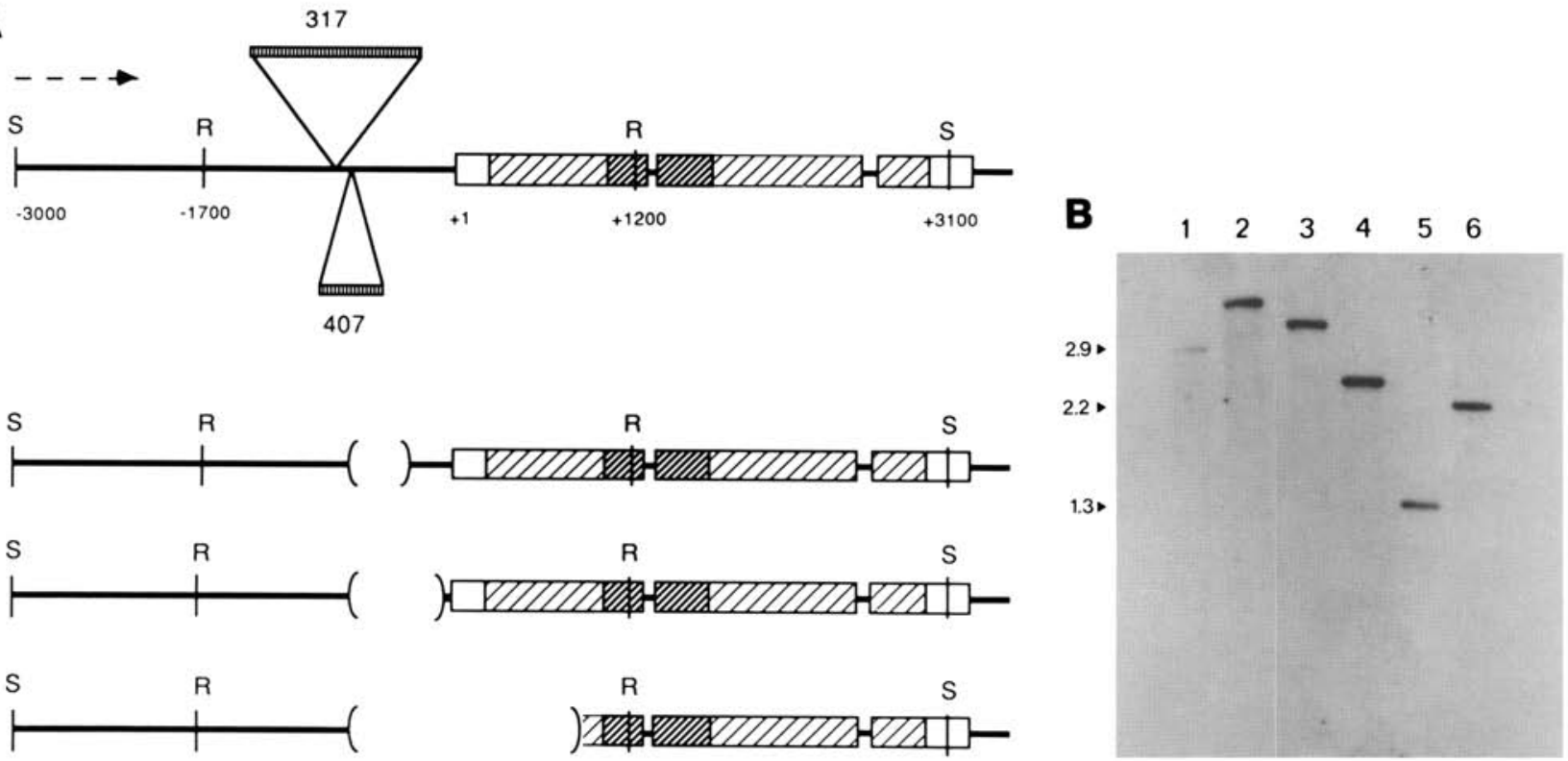

Figure 5. (A) Molecular structure of the $p q p$ locus in wild-type and mutant lines. (Top) Wild type. (Bottom) Deletions of the $p q p$ locus in mutant $(181,48)$ and phenotypeless (107) lines. Deletion breakpoints are indicated by the brackets. Transcription is from left to right. +1 indicates the transcription initiation site. (Empty boxes) Transcribed untranslated regions; (crosshatched) coding sequence; (crosshatched dark) finger region; (triangles) site and size of P-element insertions. The dashed arrow shows the 3' end of an unidentified 4-kb transcript. (R) EcoRI; (S) SalI. (B) Whole genome Southern blot analysis of wild-type, phenotypeless, and mutant lines. DNA cut by EcoRI. (Probe) EcoRI-EcoRI 2.9-kb fragment. (Lane 1) Canton S; (lane 2) line 317; (lane 3) line 407; (lane 4) line 107; (lane 5) line 48 ; (lane 6) line 181. Size of molecular mass reference markers is indicated in $\mathrm{kb}$.

two lines carrying P-element insertions and the seven lines carrying phenotypeless deletions were tested in combination with $p q p^{48}$ to determine whether they were completely wild type or weak alleles of $p q p$. No zygotic or maternal phenotype was observed in heteroallelic flies. Therefore, we assume that none of the three insertions or seven deletions affects $p q p$ expression sufficiently to produce a phenotype, even in combination with a null allele. Breakpoints of the largest phenotypeless deletion (line 107; Fig. 5A,B) were localized by sequence analysis. The $5^{\prime}$ breakpoint was located at position -715 , similarly to deletions of lines 48 and 181 . The $3^{\prime}$ breakpoint was at position -280 . We conclude from these results that sequences extending from -700 to -280 and absent in line 107 have no effect on $p q p$ expression, whereas those extending from -280 to -30 and missing in line 181 are critically required, their absence leading to a strong phenotype.

\section{Discussion}

The pqp zinc finger protein is present in the nurse cell nucleus and in the oocyte nucleus

Immunodetection experiments revealed that the distribution of the pqp protein in germ-line cells follows a complex and intriguing pattern. It is probable that the pqp protein accumulated in the oocyte nucleus at stage 7 is transported to the oocyte from the nurse cells, where it was formerly located, by the large cellular bridges con- necting the cells (Mahowald and Kambysellis 1980). Little information is available concerning the structure of the oocyte nucleus (King 1970; Mahowald and Kambysellis 1980). The spherical structures in which the pqp protein accumulates from stage 7 to stage 9 have not been described yet. Identity of the anti-pqp-revealed spherical structures with the karyosome is ruled out by the presence of two structures in some of the nuclei, but the presence of the pqp protein on the chromosomes cannot be excluded. These spherical structures are reminiscent of the sphere organelles found in the germinal vesicle of amphibians (Gall and Callan 1989). These authors detected similar structures in the oocyte of the spider Achaearanea and the cricket Acheta. The sphere organelles contain snRNPs and could be either splicing centers or spliceosome storage centers /Gall and Callan 1989|. Preliminary results using the Y12 anti-snRNP antibody, previously shown to cross-react with Drosophila snRNPs (Paterson et al. 1991), indicate the presence of snRNPs containing spheres in the Drosophila oocyte nucleus (L. Ségalat, unpubl.). Their relationship with the anti-pqp-detected spheres remains to be demonstrated.

During stages 9 and 10, the spheres gradually disappear from the oocyte staining pattern. We do not know whether they disaggregate or if they remain while the pqp protein unbinds them to be widely distributed in the nucleoplasm. During stage 9, a strong accumulation of pqp protein is detected in the nucleus of the nurse cells. This stage corresponds to the highest transcriptional activity of the nurse cells (Mahowald and Kambysellis 


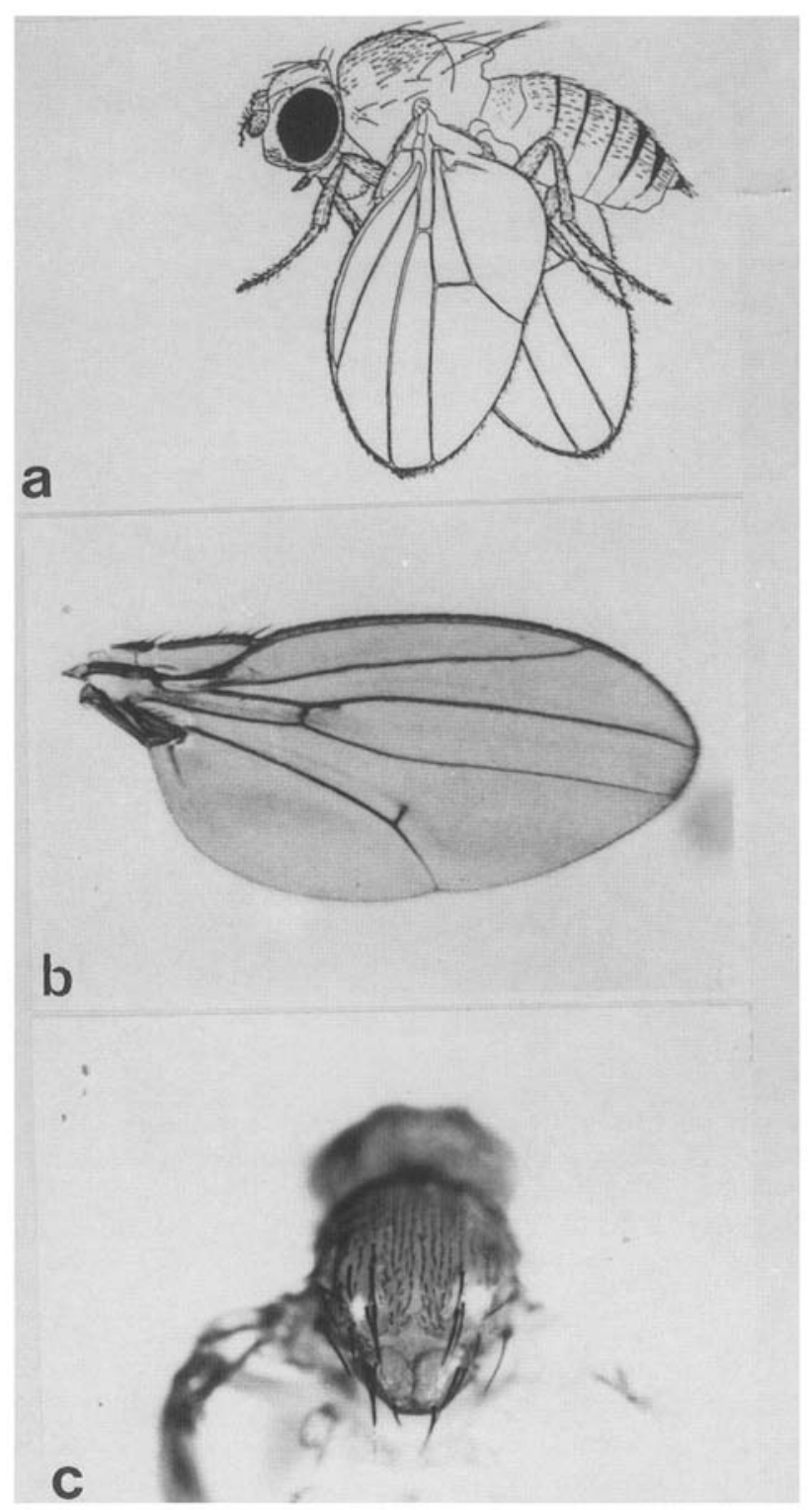

Figure 6. Zygotic phenotypes of the $p q p$ mutants. $(a)$ wings down homozygous female drawn by T.H. Morgan (1929; reproduced by courtesy of Carnegie Institution, Washington, D.C.). Wing position in $p q p^{48}$ homozygous flies is identical. $(b)$ Wing of a homozygous $p q p^{48}$ fly showing cross-vein defects. (c) Dorsal view of the thorax of a homozygous $p q p^{48}$ fly showing macrochaete duplications.

1980). The pqp protein may play the role of a nurse cell transcription factor, or it may only transit through the nurse cell nuclei before being transported to the oocyte nucleus. In the latter case, the preexisting oocyte nucleus pattern may be masked by a large influx of newly synthesized pqp protein.

\section{Does the pqp protein activate transcription in the oocyte nucleus?}

The presence of the pqp zinc finger protein, a putative transcription factor, in the oocyte nucleus raises two questions: (1) Is there any transcription in the Drosophila oocyte nucleus? (2) Does pqp regulate transcription in the oocyte nucleus?

Several lines of evidence indicate that a few genes are transcribed in the oocyte. Mahowald and Tiefert (1970) detected a short non-nucleolar uptake of $\left[{ }^{3} \mathrm{H}\right] \mathrm{dUTP}$ in stage 9 oocytes. Recently, in an enhancer-detector screen, Grossniklaus et al. (1989) found some lines in which both nurse cell nuclei and oocyte nuclei were stained and other lines in which only nurse cell nuclei were stained. As mRNA and protein encoded by the reporter gene are supposed to be identical in all lines, discrepancies between the two patterns must be explained by oocyte transcription. In addition, in situ hybridization experiments strongly suggest that the K10 and Yema genes are transcribed in the oocyte nucleus (Ait-Ahmed et al. 1987; Haenlin et al. 1987). The K10 protein is nuclear and possesses a putative DNA-binding sequence (Haenlin et al. 1987; Prost et al. 1988), but its role in oocyte transcription is unknown. It is probable that, as in any other cell type, transcription factors regulate gene expression in the oocyte. None of them has yet been formally identified.

On the other hand, the presence of a transcription factor in the oocyte nucleus does not necessarily imply that it has an influence on oocyte transcription. Most of the proteins found in the oocyte do not play a role in oogenesis but are stored for subsequent use during embryonic development. As far as the pqp protein is concerned, its presence in early embryos supports this hypothesis. Genetic and molecular identification of genes interacting with $p q p$ should indicate whether the pqp protein detected in the oocyte nucleus is or is not active.

\section{Mutagenesis strategy}

Mutagenesis screens based on PCR detection of P elements offer two advantages: (1) They require no a priori hypothesis concerning the expected phenotype and $(2)$ they allow mass screening of flies in batches (Ballinger and Benzer 1989; Kaiser and Goodwin 1990). Of 40,000 flies tested, we obtained three P-element insertions at the $p q p$ locus, five times less than would be expected theoretically if the $p q p$ locus is neither a hot spot nor a cold spot (see Materials and methods), meaning that the $p q p$ locus is a cold spot for P-element insertions. The three P-element insertions at the $p q p$ locus were phenotypeless. All three insertions were located within $110 \mathrm{bp}$ of the $5^{\prime}$-flanking region of the $p q p$ gene, thus confirming that $\mathrm{P}$ elements insert preferentially in regulatory regions (Engels 1988). P elements are known to insert preferentially in some loci and rarely in others; therefore, mutation rates of P-element-induced mutagenesis are unpredictable (Engles 1988). It must be noted, however, that most large-scale data available for P-element mutagenesis are based on phenotypic screens over an allele of a given gene and not on molecular screens (Kidwell 1987). Although there is no doubt that some loci are hot spots for P-element insertions and others are not, low 


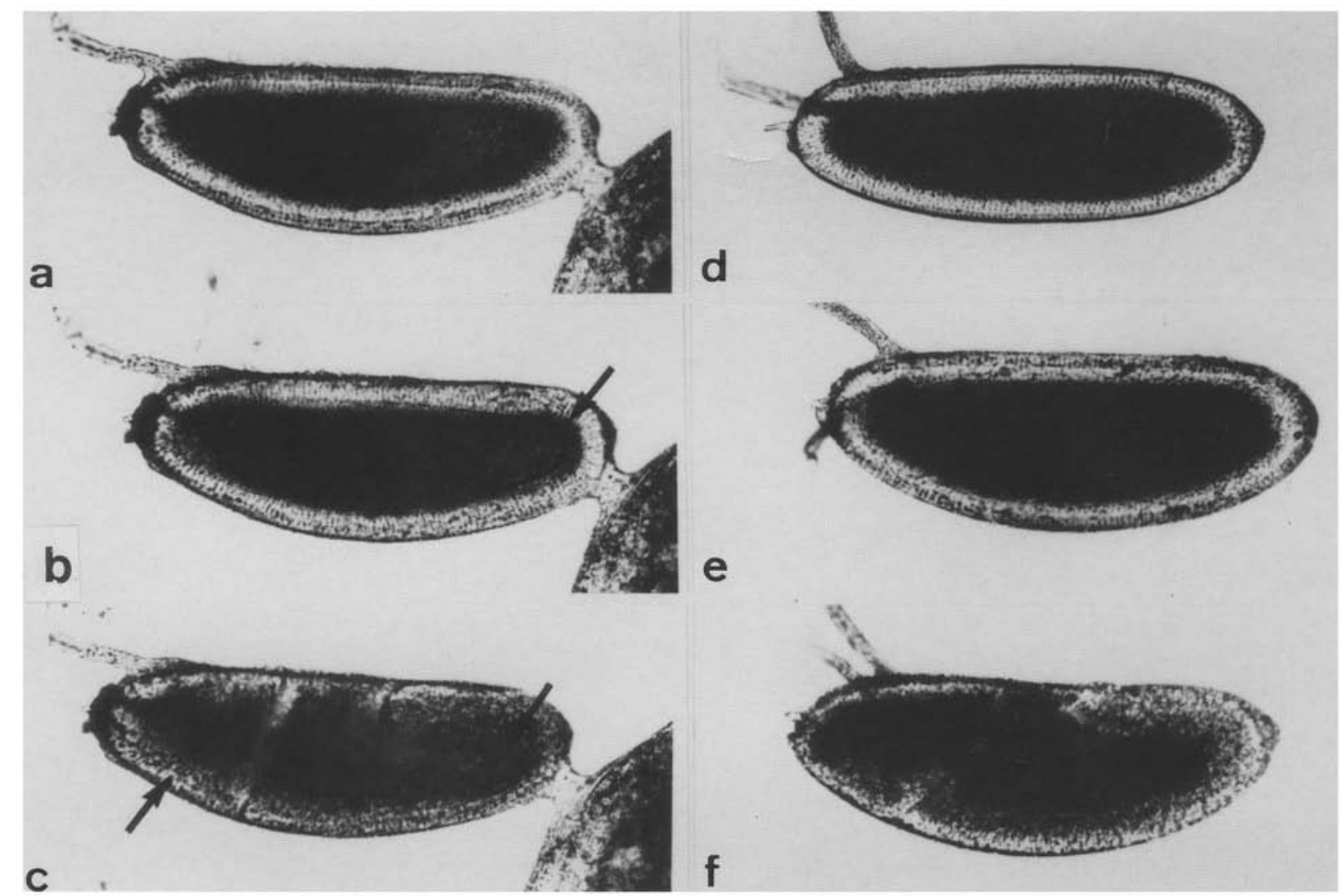

Figure 7. Maternal phenotype of the $p q p$ mutants. Early cellular blastoderm $(a, d)$, late cellular blastoderm $(b, e)$, and gastrulating $(c, f)$ embryos from homozygous $(a-c)$ and heterozygous $(d-f) p q p^{48}$ females mated to wild-type males. $(a, d, e, f)$ Development is identical to that of wild-type embryos; $(b)$ cells at the posterior end migrate laterally (arrow); $(c)$ cell migration is abnormal at the posterior end of the embryo, and the anterior midgut invagination does not form (arrows). (Left) Anterior; (top) dorsal side.

insertion frequencies observed in some phenotypic screens (Kidwell 1987) are probably the result of the combination of two negative (and maybe independent) factors: (1) low frequency of P-element insertion at a locus and (2) insertions in that locus being mostly phenotypeless. Therefore, the large discrepancies observed in mutation rates of P-element mutagenesis must be tempered by the fact that the extreme bias of P-element insertion sites within a given gene may lead to many silent insertions. Molecular screens permit circumvention of this bias. Our results show that the large P-element mutagenesis undertaken at the $p q p$ locus would have been in vain had it been based on a phenotypic screen.

The pourquoi-pas? gene is probably the wings down gene

The infrequent wing drooping phenotype of the $p q p$ mutant resembles strikingly that of one described previously and lost for many years. In a 1929 paper, T.H. Morgan described a mutant he called wings down $(w d n)$ because "wings are turned down and somewhat appressed to the sides of the body .... The wings drag on the surface over which the fly moves and, becoming wet, stick the fly to the food" (Morgan 1929). Morgan located the gene on the third chromosome at genetic position 100, which corresponds to chromosomal section 99 and is close to the location of the pqp gene (98E). This suggests strongly that $p q p$ and $w d n$ are one and the same gene. Morgan did not mention the other $p q p$ zygotic phenotypes, nor the maternal sterility, suggesting that he may have isolated a hypomorphic allele. Preliminary results of partial rescue experiments show that a small amount of pqp protein can rescue the female sterility of $p q p$ mutants but not the zygotic phenotypes (L. Ségalat, unpubl.).

\section{The pqp mutation affects different developmental pathways}

The pqp zinc finger protein is a putative transcription factor. This implies that the phenotype of $p q p$ mutants is not caused directly by the absence of a structural or catalytic cellular component but, rather, indirectly by the misexpression of $p q p$ downstream effector genes. Nevertheless, it is striking to note that the four phenotypes observed in the pqp mutants (wing position, cross-vein defects, extra bristles, and maternal sterility) belong to 
separate developmental systems: neuromuscular differentiation, intrasegmental cell identity, neurogenesis, and oogenesis, respectively. Two molecular mechanisms may underlie such a pleiotropic effect. On one hand, the pqp zinc finger protein might be a transcriptional activator/repressor of several downstream genes implicated in different developmental pathways. On the other hand, the pqp protein may be a transcription factor for a gene (or a family of genes) expressed in many different cell types (e.g., cell adhesion molecules). The $p q p$ mRNA is present in a broad range of tissues (Vincent et al. 1988), and the pqp protein binds to multiple discrete sites on the polytene chromosomes of salivary glands (L. Ségalat, unpubl.). The pqp protein may, therefore, be engaged in a complex regulatory network that is only partly revealed by the $p q p$ phenotype. Identification of genes interacting with $p q p$ should help to identify potential regulatory partners and target downstream genes.

\section{Expression of the pqp protein and pqp maternal phenotypes can be integrated into a simple model}

Ovaries of homozygous mutant females and eggs laid by these females are morphologically normal. As the pqp protein is present in both somatic and germ-line cells of the ovary, the $p q p$ maternal phenotypes might be caused by the lack of $p q p$ function in only one cell type, or in both.

The apparent absence of fertilization might be due to an abnormal vitelline membrane or micropyle, or both. It is consistent with the expression of $p q p$ in follicle cells. Dechorionated eggs do not ooze out of the vitelline membrane as they do in the $f s(2) Q / 42$ mutation, indicating that embryos from $p q p$ mothers do not lack any of the major components of the vitelline membrane (Savant and Waring 1989). Nevertheless, pqp absence in the follicle cells may lead either to a modified stoichiometry of the basic vitelline membrane components /equivalent to a hypomorphic mutation of one of them/ or to the lack of one minor component.

The most dramatic maternal effect of the $p q p$ mutation is the abnormal gastrulation that follows a more or less complete cellularization. In Drosophila, cellularization coincides with the onset of zygotic transcription (McKnight and Miller 1976). It is striking to observe that it also coincides with the appearance of developmental disorders in embryos from $p q p$ mothers. The pqp protein is a putative transcription factor located in the oocyte nucleus at the end of oogenesis. The pqp protein detected in early embryogenesis probably results from the partitioning of the maternally provided pqp protein through daughter nuclei. Hence, the link between the expression of the pqp protein and the $p q p$ maternal phenotype can be explained in a simple model in which the pqp protein is an early transcriptional activator/repressor of zygotic genes. When it is absent, embryos start a wrong developmental program that results in aberrant development and abortive embryonic growth. If this model is correct, identification of $p q p$ target genes should shed light on the poorly understood mechanism of zygotic transcriptional activation.

\section{Materials and methods}

\section{Antibodies}

The region of the pqp gene coding for amino acids 183-250 (Vincent et al. 1988) was fused in-frame downstream of the Staphylococcus aureus A protein gene in the pERAT 318 expression vector (Löwenadler et al. 1986). The fusion protein was purified by affinity chromatography on a Sepharose--IgG column (Pharmacia). Polyclonal antibodies were raised in rabbits and $\operatorname{IgG}$ were purified by chromatography on DEAE-Sepharose (Harlow and Lane 1988). The same pqp protein-coding region was fused in-frame with the Escherichia coli lacZ gene in the pUR 290 vector (Rüther and Müller-Hill 1983). The pqp- $\beta$-galactosidase fusion protein was purified by chromatography on a $p$-aminophenyl- $\beta$-D-thiogalactopyranoside (Sigma) column, covalently coupled to Affigel 10 beads (Bio-Rad), and the specific anti-pqp IgG were purified by affinity chromatography on this matrix.

The Ajl antibody was a gift from $\mathrm{H}$. Saumweber (Köln University, Germany). The Y12 anti-snRNPs antibody was a gift from G. Cathala and C. Brunel (CNRS URA 1191, Montpellier, France).

\section{Immunostaining}

Ovaries were dissected in phosphate-buffered saline (PBS), fixed [15 min, room temperature (RT)] with $0.4 \%$ paraformaldehyde in PBS, and washed extensively with PBS (RT). To reduce staining background, ovaries were incubated ( $1 \mathrm{hr}, \mathrm{RT})$ in PBS with $1 \%$ BSA and $0.1 \%$ Triton $\mathrm{X}-100$ and treated with the Vector blocking kit. Ovaries were incubated (overnight, $4^{\circ} \mathrm{C}$ ) with the primary antibody diluted in PBS (anti-pqp 1/200), washed $(2 \mathrm{hr}$, RT) with PBS, and incubated further $(1 \mathrm{hr}, \mathrm{RT})$ with an antirabbit or anti-mouse biotinylated secondary antibody (Vector) preadsorbed $(\geqslant 1 \mathrm{hr}, \mathrm{RT})$ with ovaries and diluted $1 / 2000$ in incubation buffer. Ovaries were then washed ( $2 \mathrm{hr}, \mathrm{RT})$ with PBS and incubated (20 min, RT) with the ABC-peroxidase complex from the Vectastain kit (Vector) in PBS with $0.1 \%$ Tween 20. After washing ( $30 \mathrm{~min}, \mathrm{RT})$, ovaries were stained in PBS

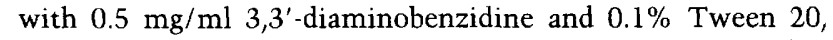
washed with PBS (10 min, RT), and mounted in $60 \%$ glycerol in PBS. Control incubations without primary antibody were performed in parallel. Slides were observed with a Leitz microscope using Nomarski lenses and photographed using Technical Pan (Kodak) film. Developmental stages of oogenesis are given according to Mahowald (Mahowald and Kambysellis 1980). Embryos were stained and mounted following standard protocols (Ashburner 1989b). For phenotypic descriptions, living embryos were observed and photographed in transmitted light according to standard protocols (Schüpbach and Wieschaus 1989).

D. melanogaster stocks, mutagenesis procedures, and PCR screen

The Canton S stock was used as a wild-type reference. Other stocks cited were kindly provided by members of $D$. Anxolabehere's laboratory (Institut Jacques Monod, Paris, France). P[ry+ $\Delta 2-3](99 \mathrm{~B})$ and Birm 2 chromosomes are described in Engels (1988).

For P-element insertional mutagenesis, males or virgin females were mated to $\mathrm{P}[r y+\Delta 2-3](99 \mathrm{~B})$ flies at $18^{\circ} \mathrm{C}$ to avoid pupal lethality. $\mathrm{F}_{1}$ males Birm $2 /+$; $\mathrm{P}[\mathrm{ry}+\Delta 2-3](99 \mathrm{~B}) /+{ }^{*}$ were 
mated to Birm2/Birm2; TM6/Sb virgin females at $25^{\circ} \mathrm{C}$. Southern blot analysis revealed that this genetic combination yields an average of four P-element insertions per third chromosome per gamete. Because $>95 \%$ of Birm $2 / \mathrm{P}[r y+\Delta 2-3]$ (99B) flies suffer pupal lethality at $25^{\circ} \mathrm{C}$, most of the progeny was transposase-free. Females were collected once a day, gathered in batches of 100 , and mated to TM3/TM1 males. After 3-5 days, the eggs of each batch were collected overnight. DNA extraction and PCR reactions were performed according to Kaiser and Goodwin (1990). PCR products were separated on a $1 \%$ agarose gel, blotted on Schleicher and Schüll BA85 nitrocellulose filters, and hybridized using standard protocols (Maniatis et al. 1989) with the $p q p \leqslant$ llI-Sall $6.1-\mathrm{kb}$ fragment labeled by nick translation. When a lane was positive, parents were divided into groups of 10 and the same procedure was applied. The last round was performed directly on individual females. Primers were the 31 bp repeat of P-element wings and two pqp-specific 30-mer beginning at positions +3220 and +280 . Control experiments to estimate the sensitivity of the method were done by introducing a single transgenic fly carrying an extra copy of the $p q p$ gene into a batch of candidate flies and testing whether this fly can be identified using different sets of primers. Results showed that the procedure was sensitive enough to detect a P-element insertion $\leqslant 3 \mathrm{~kb}$ from the pqp primer. Because two pqp primers were used, we were able to detect a P-element insertion approximately between positions +3200 and -2800 .

First, 10,000 flies were screened according to the following calculations: Considering that the third chromosome is $\sim 60,000 \mathrm{~kb}$ (Ashburner 1989a) and that we scanned a 6-kb region with our primers, the mean expected number of $P$ insertions in the scanned region (if $p q p$ is neither a hot spot nor a cold spot for $\mathrm{P}$ insertions) is 1 per 10,000 insertions on the third chromosome (which is $1 / 2500$ flies; see above). When no P-element insertion was detected in the first series of 10,000 flies, 30,000 more were screened (total: 40,000 flies or 160,000 insertions on the third chromosomel.

For excision-induced deletions, males or virgin females of the line 407 were mated to $\mathrm{P}[$ ry $+\Delta 2-3](99 \mathrm{~B})$ flies at $22^{\circ} \mathrm{C}$. F1 males were mated to TM6/1(3) virgin females and F2 407*/TM6 males were crossed individually to $\mathrm{TM} 3 / 1|3|$ females to establish lines. Lines were tested by Southern blot analysis using standard protocols (Maniatis et al. 1989) with the pqp SalI-SalI 6.1$\mathrm{kb}$ fragment labeled by nick translation.

\section{Transgenic flies}

DNA manipulations were performed following standard protocols (Maniatis et al. 1989). To clone the pqp wild-type copy, the SalI-Sall 6.1-kb fragment was cut from a subclone of $\lambda$ Dmsryhl 8 (Vincent et al. 1988) and inserted in the Sall site of the pDM23 transformation vector (Mismer and Rubin 1987). For cloning of the mutated pqp copy (deleted from +1 to +300 ), two cohesive fragments extending from -1700 to -1 and +301 to +1200 were produced by PCR. They were joined by ligation to form a 2.6-kb EcoRI-EcoRI fragment. The wild-type $2.9-\mathrm{kb}$ $E c o$ RI-EcoRI fragment was then replaced by the mutated version to form a mutated 5.8-kb Sall-Sall fragment that was introduced into the pDM23 vector. Both plasmids were purified on a $\mathrm{CsCl}$ gradient and injected in rosy ${ }^{506}$ embryos using standard transformation protocols (Rubin and Spradling 1982). For each construct, two lines carrying the transgene on the second chromosome were kept.

\section{Acknowledgments}

We thank P. Feynerol and K. Taalba for excellent technical as- sistance and R. Schwartzman for photographic work. This work was supported by a fellowship from the Ministère de la Recherche et de la Technologie to L.S. and by grants from the Centre National de la Recherche Scientifique, the Association pour la Recherche sur le Cancer, the Fondation pour la Recherche Médicale and the Ligue Nationale Contre le Cancer to J.A.L.

The publication costs of this article were defrayed in part by payment of page charges. This article must therefore be hereby marked "advertisement" in accordance with 18 USC section 1734 solely to indicate this fact.

\section{References}

Aït-Ahmed, O., M. Thomas-Cavallin, and R. Rosset. 1987. Isolation and characterization of a region of the Drosophila genome which contains a cluster of differentially-expressed maternal genes (yema gene region). Dev. Biol. 122: 153-162.

Ashburner, M. 1989a. Drosophila: A laboratory handbook. Cold Spring Harbor Laboratory Press, Cold Spring Harbor, New York.

1989b. Drosophila: A laboratory manual. Cold Spring Harbor Laboratory Press, Cold Spring Harbor, New York.

Ballinger, D.G. and S. Benzer. 1989. Targeted gene mutations in Drosophila. Proc. Natl. Acad. Sci. 86: 9402-9406.

Edgar, B.A. and G. Schubiger. 1986. Parameters controlling transcriptional activation during early Drosophila development. Cell 44: 871-877.

Engels, W.R. 1988. P elements in Drosophila. In Mobile DNA (ed. D. Berg and M. Howe), pp. 437-484. ASM Publications, Washington, D.C.

Evans, R.M. and S.M. Hollenberg. 1988. Zinc fingers: Gilt by association. Cell 52: 1-3.

Gall, J. and H.G. Callan. 1989. The sphere organelle contains small nuclear ribonucleoproteins. Proc. Natl. Acad. Sci. 86: $6635-6639$.

Grossniklaus, U., H.J. Bellen, C. Wilson, and W.J. Gehring. 1989. P-element -mediated enhancer detection applied to the study of oogenesis in Drosophila. Development 107: 189 200.

Gurdon, J.B. 1992. The generation of diversity and pattern in animal development. Cell 68: 185-199.

Haenlin, M., C. Roos, A. Cassab, and E. Mohier. 1987. Oocytespecific transcription of $f_{S}(1)$ K10: A Drosophila gene affecting dorso-ventral developmental polarity. $E M B O$ I. 6: 801807

Harlow, E. and D. Lane. 1988. Antibodies: A laboratory manual. Cold Spring Harbor Laboratory, Cold Spring Harbor, New York.

Harrison, S.D. and A.A. Travers. 1990. The tramtrack gene encodes a Drosophila finger protein that interacts with the $f t z$ transcriptional regulatory region and shows a novel embryonic expression pattern. EMBO /. 9: 207-216.

Kaiser, K. and S.F. Goodwin. 1990. Site-selected transposon mutagenesis of Drosophila. Proc. Natl. Acad. Sci. 87: 16861690.

Kidwell, M.G. 1987. A survey of success rates using P element mutagenesis in Drosophila melanogaster. Drosophila Inf. Service 66: 81-86.

King, R.C. 1970. Ovarian development in Drosophila melanogaster. Academic Press, New York.

Lesley Brown, I., S. Sonoda, H. Ueda, M. Scott, and C. Wu. 1991. Repression of the Drosophila fushi tarazu (ftz) segmentation gene. $E M B O$ I. 10: 665-674.

Löwenadler, B., B. Nilsson, L. Abrahmsén, T. Moks, L. Ljungqvist, E. Holgrem, S. Paleus, S. Josephson, L. Philipson, 
and M. Uhlén. 1986. Production of specific antibodies against protein A fusion proteins. EMBO I. 5: 2393-2398.

Mahowald, A.P. and M. Tiefert. 1970. Fine structural changes in the Drosophila oocyte nucleus during a short period of RNA synthesis. Wilhelm Roux's Arch. Dev. Biol. 165: 8-25.

Mahowald, A.P. and M.P. Kambysellis. 1980. Oogenesis. In The genetics and biology of Drosophila (ed. M. Ashburner and T.R.F. Wright), vol. 2, pp. 141-224. Academic Press, New York.

Maniatis, T., E.F. Fritsch, and J. Sambrook. 1989. Molecular cloning: A laboratory manual. Cold Spring Harbor Laboratory Press, Cold Spring Harbor, New York.

McKnight, S.L. and O.L.J. Miller. 1976. Ultrastructural patterns of RNA synthesis during early embryogenesis in Drosophila melanogaster. Cell 8: 305-319.

Mismer, D. and G.M. Rubin. 1987. Analysis of the promoter of the ninaE opsin gene in Drosophila melanogaster. Genetics 116: $566-578$.

Morgan, T.H. 1929. Data relating to six mutants of Drosophila. Carnegie Inst. Wash. Publ. 399: 171-179.

Paterson, T., J.D. Beggs, D.J. Finnegan, and R. Lührmann. 1991. Polypeptide components of Drosophila small nuclear ribonucleoprotein particles. Nucleic Acids Res. 19: 5877-5882.

Prost, E., F. Deryckere, C. Roos, M. Haenlin, V. Pantesco, and E. Mohier. 1988. Role of the oocyte nucleus in determination of the dorsoventral polarity of Drosophila as revealed by molecular analysis of the K10 gene. Genes \& Dev. 2: 891-900.

Robertson, H.M, C.R. Preston, R.W. Phillis, D.M. JohnsonSchlitz, W.K. Benz, and W.R. Engels. 1988. A stable genomic source of $\mathrm{P}$ element transposase in Drosophila melanogaster. Genetics 118: 461-470.

Rubin, G.M. and A.C. Spradling. 1982. Genetic transformation of Drosophila with transposable element vectors. Science 218: 348-353.

Rüther, U. and B. Müller-Hill. 1983. Easy identification of cDNA clones. EMBO I. 2: 1791-1794.

St. Johnston, D. and C. Nüsslein-Volhard. 1992. The origin of pattern and polarity in the Drosophila embryo. Cell 68: 201291.

Saumweber, H., P. Symmons, R. Kabish, H. Will, and F. Bonnhoeffer. 1980. Monoclonal antibodies against chromosomal proteins of Drosophila melanogaster. Chromosoma 80: $253-275$.

Savant, S.S. and G.L. Waring. 1989. Molecular analysis and rescue of a vitelline membrane mutant in Drosophila. Dev. Biol. 135: 43-52.

Schüpbach, T. and E. Wieschaus. 1989. Female sterile mutations on the second chromosome of Drosophila melanogaster. I. Maternal effect mutations. Genetics 121: 101-117.

Vincent, A., J. Kejzlarovà-Lepesant, L. Ségalat, C. Yanicostas, and J.-A. Lepesant. 1988. sry h-1, a new Drosophila multifingered protein gene showing a maternal and zygotic expression. Mol. Cell. Biol. 8: 4459-4468. 


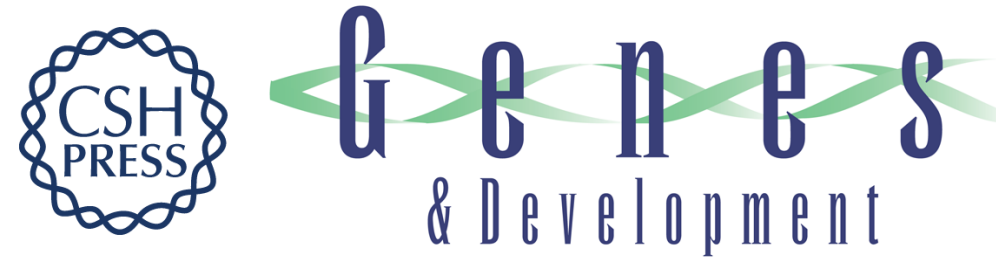

\section{The Drosophila pourquoi-pas?/wings-down zinc finger protein: oocyte nucleus localization and embryonic requirement.}

L Ségalat, R Perichon, J P Bouly, et al.

Genes Dev. 1992, 6:

Access the most recent version at doi:10.1101/gad.6.6.1019

References This article cites 27 articles, 9 of which can be accessed free at:

http://genesdev.cshlp.org/content/6/6/1019.full.html\#ref-list-1

License

Email Alerting

Service

Receive free email alerts when new articles cite this article - sign up in the box at the top right corner of the article or click here.

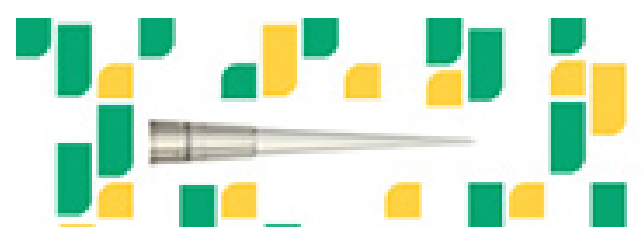

Focused on your science. 